\title{
ORIGINAL
}

\section{SALUD E INCIDENCIA Y DIFERENCIAS EN VULNERABILIDAD TERRITORIAL DE LA CIUDAD DE MADRID} \author{
e Isaac Fernandez del Pozo (4) \\ (1) Departamento de Estudios Territoriales. Ayuntamiento de Madrid. Madrid. España. \\ (2) Departamento de Salud Pública y Materno-Infantil. Universidad Complutense. Madrid. España. \\ (3) Medicina Preventiva y Salud Pública. Universidad Rey Juan Carlos. Móstoles. España. \\ (4) Departamento de Matemática e Informática. Universidad Politécnica. Madrid. España.
}

María Mercedes Esteban y Peña (1), Elena Fernández Velasco (1), Rodrigo Jiménez García (2), Valentín Hernández Barrera (3)

Los autores declaran que no existe conflicto de intereses.

\section{RESUMEN}

Fundamentos: La territorialidad se configura como un eje importante de desigualdad. El objetivo de este estudio fue determinar el nivel de asociación entre territorio y vulnerabilidad, concretando propuestas de intervención territorial mediante indicadores clave socio-sanitarios.

Métodos: Se realizó un estudio transversal analítico, que combinó el análisis de la Encuesta de Salud de la Ciudad de Madrid de 2017 ( $\mathrm{n}=9.513$ ) y el indicador de vulnerabilidad-ranking del mismo año $(\mathrm{n}=2.780 .197$, población de la ciudad de Madrid en 2017, procedente del Banco de Datos del Ayuntamiento de Madrid), elaborado con la técnica de análisis jerárquico. Se incluyeron variables sociodemográficas y de salud, como autopercepción del estado de salud, calidad de vida en relación con la salud, consumo de tabaco, alcohol, obesidad, sedentarismo y salud mental (GHQ-12). Las relaciones se valoraron con DE, IC 95\%, correlación de Spearman, coeficientes B y $\beta$ de regresión lineal múltiple y la técnica de pares de puntos.

Resultados: Los vínculos entre salud y vulnerabilidad fueron: en salud-autopercibida y CVRS, como variables globales de salud, $\mathrm{p}<0,01$ para las mujeres con territorio y vulnerabilidad; en sedentarismo para ambos sexos, se interpretó con el $60-80 \%$ por el territorio y vulnerabilidad; la obesidad se vinculó levemente con el territorio en las mujeres y explicó el $77 \%$; la salud mental no se relacionó territorialmente para el conjunto, pero sí de forma significativa en las mujeres, por el $64 \%$ de la varianza; en tabaco hubo vínculo significativo territorial en hombres y vulnerabilidad en el $57 \%$; finalmente, el alcohol tuvo una diferencia significativa en hombres a nivel territorial y explicó en el $72 \%$ en ambos sexos, de forma invertida.

Conclusiones: Los resultados avalan el comportamiento de los indicadores globales y específicos de salud con la vulnerabilidad, con una desagregación por sexos, lo cual permitirá una planificación adaptada al territorio.

Palabras clave: Determinantes de salud, Desigualdades sociales, Contexto, Obesidad, Sedentarismo, Salud mental, Barrios.

Correspondencia:

Maria Mercedes Esteban y Peña

Departamento de Estudios

Área de Coordinación Territorial

C/ Montalbán, 1

28014 Madrid, España

estebanpm@madrid.es

\section{ABSTRACT \\ Health, incidence and differences in the territorial vulnerability city of Madrid}

Background: Territoriality is configured as an important axis of inequality. The objective of this study was to determine the level of association between territory and vulnerability, specifying proposals for territorial intervention using key socio-health indicators.

Methods: Analytical cross-sectional study, which combined the analysis of the Health Survey Madrid City $2017(\mathrm{n}=9,513)$ and the vulnerability-ranking indicator of the same year $(\mathrm{n}=2,780,197)$, population of the city of Madrid in 2017, from the Data Bank of the Madrid City Council), prepared with the hierarchical analysis technique. Sociodemographic and health variables were included, such as self-perception of health status, quality of life in relation to health, tobacco consumption, alcohol, obesity, sedentary lifestyle and mental health (GHQ-12). The relationships were assessed with DE, 95\% CI, Spearman correlation, $B$ and $\beta$ coefficients of multiple linear regression and the pair-point technique.

Results: The links between health and vulnerability were: in health-self-perceived and HRQL, as global health variables, $\mathrm{p}<0.01$ for women with territory and vulnerability; sedentary lifestyle for both sexes, was interpreted with $60-80 \%$ by territory and vulnerability; obesity was slightly linked to the territory in women and explained $77 \%$; mental health was not territorially related for the group, but it was significantly related to women, due to $64 \%$ of the variance; in tobacco there was a significant territorial link in men and vulnerability in 57\%; finally, alcohol had a significant difference in men at the territorial level and explained in $72 \%$ in both sexes, inverted.

Conclusions: The results support the behavior of global and specific health indicators with vulnerability, with a disaggregation by sex, which will allow planning adapted to the territory.

Key words: Health determinants, Social inequalities, Context, Obesity, Sedentary lifestyle, Mental health, Neighborhoods.

Cita sugerida: Esteban y Peña MM, Fernandez Velasco E, Jiménez García R, Hernández Barrera V, Fernandez del Pozo I. Salud e incidencia y diferencias en vulnerabilidad territorial de la ciudad de Madrid. Rev Esp Salud Pública. 2020; $94: 7$ de abril e202004020 


\section{INTRODUCCIÓN}

En la definición de los ejes que componen la desigualdad social, el territorio o el contexto, como lugar donde se vive, es uno de ellos, situado al mismo nivel que el género, la edad, las relaciones de poder o la clase social. Por tanto, el concepto de territorialidad es un factor de desigualdad y vulnerabilidad de las poblaciones, incorporado al marco conceptual ${ }^{(1,2,3)}$. La vinculación entre vulnerabilidad y territorio se plantea como un paradigma de para quién y dónde intervenir. No solo se refiere a que las personas vulnerables tengan peor salud, sino que nos enfocamos hacia problemas concretos, a través de un grupo de indicadores clave de morbilidad ${ }^{(4,5,6)}$ como ejes fundamentales de la salud física, psíquica, social o funcional. Incluimos los denominados "big four" o cuatro grandes problemas ("smoking", "drinking", "diet", "exercise", es decir, tabaco, bebida, dieta, ejercicio) que la epidemiología social actual configura y aborda planteados en diferentes esferas y marcos teóricos ${ }^{(3)}$.

La territorialidad posee múltiples espectros, tanto en su continente como en su contenido. Se trata de un concepto complejo y compuesto, que va más allá de lo puramente geográfico, distinguiendo entre área (zona espacial) e interior (composición), con diversidades migratorias, culturales, políticas o económicas ${ }^{(2,4)}$. Con estas premisas, se llegó a la conocida como teoría de la falacia ecológica ${ }^{(7)}$. El individuo no tiene siempre lo que el colectivo aporta. Entre donde se vive y el interior, hay un conjunto de diversidades que pueden explicar la salud por vías causales, culturales, económicas o migratorias ${ }^{(5)}$.

Una de las razones de este estudio, realizado en la ciudad de Madrid, fue justificar con fundamentos científicos el pragmatismo de la intervención, desde la organización administrativa próxima -o ya diseñada- en la planificación $y$, por tanto, la solución de los problemas reales en determinadas áreas geográficas, para intervenir desde el colectivo a lo más común (G. Rose, $1992)^{(8)}$. En definitiva, mostrar los principales problemas de salud para poder actuar sobre ellos.

La novedad viene marcada por el enfoque de estudio desde la morbilidad en lugar de la mortalidad tradicional ${ }^{(5)}$. La relación de estudios previos en este sentido es escasa. Se han realizado diferentes trabajos, como los de Steinar ${ }^{(9)}$ en Noruega o Zang ${ }^{(10)}$ en EE.UU, abordando problemas aislados como la obesidad, y delimitados geográficamente. Estos estudios marcan la relación de esta con la vulnerabilidad, o con perfiles socioeconómicos, lo que permite la intervención dirigida mediante "proporcionalidad inversa" para la acción comunitaria en el campo de la prevención y promoción de la sa$\operatorname{lud}^{(7)}$. También, analizando problemas de violencia, asociados con los niveles socioeconó$\operatorname{micos}^{(6)}$, o los que vinculan vecindario de mala construcción y posibilidad de depresión ${ }^{(11)}$, o peor salud mental con zonas más vulnerables, o mayor carga de enfermedad crónica frente a enfermedad infecciosa descrita en el pasado ${ }^{(3)}$.

En nuestro ámbito geográfico próximo (la ciudad de Madrid y sus distritos), el proyecto MEDEA, con sus diversos desarrollos y desagregaciones territoriales en secciones censales, permite aproximarse a la valoración de los problemas de salud mediante los determinantes sociales o económicos de la misma ${ }^{(12)}$.

Madrid presenta zonas desiguales, y desde el Área de Gobierno de Coordinación Territorial nos aproximamos mediante un indicador de vulnerabilidad, el cual permite configurar y clasificar estos espacios en la ciudad ${ }^{(13)}$. Gracias a este índice, se describen las distribuciones de los principales problemas de salud y se trata de establecer el nivel de asociación. Es pues una herramienta perfecta que justifica la planificación de una intervención ${ }^{(14)}$. 
El objetivo de este trabajo fue determinar el nivel de asociación entre el territorio, a través de sus distritos, respecto a la morbilidad física, psíquica y social, así como la distribución de hábitos y estilos de vida saludables en una gran ciudad como Madrid frente a vulnerabilidad, para justificar así propuestas de intervención territorial a través de un grupo clave de indicadores socio-sanitarios.

\section{MATERIAL Y METODOS}

Diseño y tipo de estudio. Se realizó un estudio transversal analítico, combinando el análisis entre los resultados de un estudio descriptivo, la Encuesta de Salud de la ciudad de Madrid de 2017, y el indicador de vulnerabilidad-ranking de 2017, elaborado por análisis jerárquico (AHP) y procedente de fuentes secundarias ${ }^{(12)}$. La Encuesta de Salud tuvo una muestra representativa de la población no institucionalizada de la ciudad de Madrid de 8.845 entrevistas y en torno a 400 encuestas por distrito. El trabajo de campo se realizó entre octubre y diciembre de 2017 , con un error de muestreo de $\pm 1 \%$ en la ciudad de Madrid. La información se recopiló con un cuestionario estructurado ${ }^{(14)}$.

Variables. Las variables sociodemográficas de edad, sexo e inmigración que componen el indicador de vulnerabilidad provenían de fuentes secundarias de Estadística Municipal del Ayuntamiento de Madrid. Los distritos administrativos que componen la Ciudad son 21: Centro, Arganzuela, Retiro, Salamanca, Chamartín, Tetuán, Chamberí, Fuencarral-El Pardo, Moncloa-Aravaca, Latina, Carabanchel, Usera, Puente de Vallecas, Moratalaz, Ciudad Lineal, Hortaleza, Villaverde, Villa de Vallecas, Vicálvaro, San Blas-Canillejas y Barajas.

El Índice de vulnerabilidad-ranking de 2017 es un indicador sintético elaborado para barrios y distritos de la ciudad de Madrid con las siguientes variables: tasa de inmigrantes, esperanza media de vida al nacer, nivel de estudios, número de habitantes, renta media por hogar, tasa de paro absoluto, tasa de paro de mayores 45 años, tasa de paro de personas sin prestación, valor catastral de las viviendas, tasa de demanda de dependientes, familias perceptoras de renta mínima, tasa de Servicio de Ayuda a Domicilio (SAD) a dependientes y tasa de tele asistencia a dependientes. El índice se confecciona aplicando previamente un factor de ponderación establecido por la técnica del análisis jerárquico (AHP). El indicador final resulta de la suma de cada uno de los valores previamente ponderados. Los datos proceden de la fuente secundaria de Estadística del Ayuntamiento de Madrid ${ }^{(13,15)}$. La vulnerabilidad queda descrita en cada uno de los 21 distritos con un valor de resultado, que se ordena con respecto al resto de los valores, dando como resultado entonces el denominado ranking.

Se incluyeron también variables de salud procedentes de la Encuesta de Salud de la ciudad de Madrid de $2017^{(16)}$, que contienen algunas autoreferidas y que serían: autopercepción del estado de salud (grupo de muy bueno y bueno), calidad de vida en relación con la salud (medido por las láminas $\mathrm{COOP} / \mathrm{WONCA}^{(17)}$, consumo de tabaco (si fumaba diariamente), consumo de alcohol (si consumía cuatro o más veces a la semana), autoreferencia de obesidad (IMC mayor de 30), presencia de sedentarismo (no hacía ejercicio), salud mental recogida a través del Cuestionario de Salud General de 12 ítems (GHQ-12, cada uno de los ítems evalúa un posible problema mental en las últimas semanas, utilizando una escala tipo Likert de 4 puntos en la que 3 puntos o más significa caso de alto riesgo). El cuestionario lo completaron por vía telefónica personas menores de 65 años ${ }^{(16,18,19)}$.

Las láminas COOP / WONCA de 9 ítems, validadas en español, se utilizaron para medir 
la CVRS. Cuanto más alta es la puntuación, peor es la percepción de la CVRS en las 2 semanas anteriores. Los resultados se expresan a su vez en un "índice resumen". El cuestionario está representado por un solo factor, lo que significa que se puede usar un índice COOP / WONCA global obtenido de la suma de los ítems respectivos ${ }^{(20,21,22)}$.

Análisis estadístico. Se describieron las variables de salud clasificándolas por niveles de vulnerabilidad, usando valores medios, porcentajes, desviaciones estándar (DE) e intervalos de confianza (IC 95\%). Secundariamente, se presentaron las correlaciones entre los valores de vulnerabilidad y los de las variables de salud y hábitos-estilos de vida, utilizando el estadístico de correlación lineal de Spearman, entre las diferentes variables. La significación se estableció en $\mathrm{p} \leq 0,05$. Finalmente, se elaboró un modelo multivariante de regresión lineal, empleando la vulnerabilidad como variable dependiente, $y$ las de salud (autopercepción del estado de salud, calidad de vida, salud mental, consumo de tabaco, alcohol, sedentarismo y obesidad) como independientes, marcando la significación en $p \leq 0,05$. Para valorar la aproximación al modelo lineal, se utilizaron los estadísticos de $\mathrm{B}$ y $\beta$ que miden la tendencia de la asociación con el punto de corte en el modelo lineal y la tangente, respectivamente, así como la varianza explicada por el modelo. Para la representación gráfica se empleó la técnica de pares de puntos de las diferentes variables independientes, calculando cada una de las derivadas y estableciendo una relación de orden entre dichas rectas. Se comprobó cómo se relacionaba, de forma ordenada, la vulnerabilidad con cada una de las variables, estableciendo un criterio matemático para comparar de forma estructurada dichas relaciones.

Todos los análisis se realizaron estratificados por sexo y se utilizaron los programas estadísticos Excel, SPSS 17.0 y Desmos.com.

\section{RESULTADOS}

En la tabla 1 se describen las variables de salud, autopercepción de salud, CVRS y salud mental, estudiadas en cada uno de los 21 distritos, ordenados según valores de vulnerabilidad y mostrando la diferencia con su significación entre los distritos más y menos vulnerables. En la tabla 2, se exponen el consumo de tabaco, de alcohol, la obesidad y el sedentarismo, desagregados por sexo.

Las diferencias territoriales fueron significativas entre el distrito más y menos vulnerable para el valor global de las variables estudiadas de ambos sexos, con una $\mathrm{p} \leq 0,05$ en los siguientes indicadores: autopercepción del estado de salud (buena + muy buena), CVRS, consumo de tabaco y de alcohol, obesidad y sedentarismo. No fue significativa la diferencia entre el distrito más y menos vulnerable en la prevalencia referida a tener una posible enfermedad mental.

Desagregando por sexo, como elemento clave diferenciador, las diferencias fueron significativas entre hombres y mujeres en todo este grupo de variables seleccionadas para el conjunto de la ciudad. Las discrepancias entre territorio de distrito más vulnerable y menos, fue significativa con $\mathrm{p} \leq 0,05$ para las mujeres en autopercepción del estado de salud (muy bueno + bueno) y en calidad de vida en relación con la salud. En salud mental no hubo diferencias significativas en prevalencia territorial, ni para hombres ni para mujeres. En consumo de tabaco, la diferencia entre el distrito más y menos vulnerable fue mayor en los hombres, así como en el consumo de alcohol, con prevalencia inversa al indicador de vulnerabilidad. En el caso del sedentarismo, hubo diferencia territorial por distritos tanto en hombres como en mujeres y, en el caso de la obesidad, hubo diferencia entre los distritos más y menos vulnerables para las mujeres, aunque no entre los más extremos. 


\begin{tabular}{|c|c|c|c|c|c|c|c|c|c|c|c|c|c|c|c|c|c|c|c|c|c|c|c|c|c|}
\hline \multirow{6}{*}{ 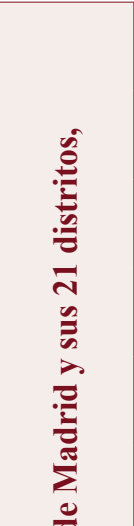 } & \multirow{6}{*}{ 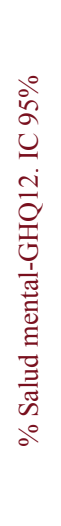 } & \multirow{3}{*}{$\cdot \stackrel{\vec{\omega}}{\vec{\Xi}}$} & $\begin{array}{c}\tilde{\delta} \\
\dot{\delta} \\
\tilde{i}\end{array}$ & $\begin{array}{l}q \\
\dot{m}\end{array}$ & $\frac{n}{2}$ & $\begin{array}{l}\hat{n} \\
\text { nn }\end{array}$ & 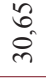 & $\frac{\forall}{m}$ & $\mid \begin{array}{l}\tilde{C} \\
\sigma \\
\forall \\
\forall\end{array}$ & $\begin{array}{l}1 \\
\text { n. } \\
\text { nn }\end{array}$ & $\begin{array}{l}n \\
6 \\
\dot{m} \\
m\end{array}$ & $\begin{array}{l}\bar{Z} \\
\dot{m}\end{array}$ & $\begin{array}{l}\vec{b} \\
\stackrel{0}{n}\end{array}$ & $\begin{array}{l}\stackrel{\infty}{+} \\
\text { î } \\
\text { in }\end{array}$ & $\begin{array}{l}\stackrel{尺}{\hat{N}} \\
\hat{N}\end{array}$ & $\begin{array}{c}\hat{n} \\
\tilde{v}\end{array}$ & $\begin{array}{l}\infty \\
\infty \\
\infty \\
\infty \\
\sim\end{array}$ & 을 & $\mid \begin{array}{l}\infty \\
\tilde{\lambda} \\
\hat{\lambda}\end{array}$ & $\begin{array}{l}\tilde{s} \\
\hat{\sigma} \\
\sim\end{array}$ & $\begin{array}{l}\tilde{n} \\
\tilde{n}\end{array}$ & $\begin{array}{l}\overrightarrow{0} \\
\dot{0} \\
0\end{array}$ & $\begin{array}{l}\hat{\text { v }} \\
\text { ה }\end{array}$ & 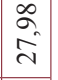 & \\
\hline & & & $\begin{array}{l}\hat{0} \\
\hat{\jmath}\end{array}$ & $\begin{array}{l}\circ \\
\stackrel{1}{1} \\
\text { (n) }\end{array}$ & $\begin{array}{l}\infty \\
\stackrel{\infty}{0} \\
\infty \\
\sim\end{array}$ & $\frac{\tilde{\sigma}}{\vec{\lambda}}$ & $\begin{array}{l}\text { กิ } \\
\text { हิ }\end{array}$ & $\begin{array}{l}\vec{n} \\
\hat{n}\end{array}$ & $\begin{array}{l}\hat{0} \\
\hat{\jmath}\end{array}$ & $\frac{N}{\approx}$ & $\frac{m}{m}$ & $\begin{array}{l}\infty \\
n \\
\tilde{n}\end{array}$ & $\begin{array}{l}\text { mे } \\
\text { 2. }\end{array}$ & 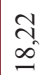 & $\begin{array}{l}g_{+} \\
\stackrel{s}{z}\end{array}$ & $\frac{尺}{\stackrel{2}{r}}$ & $\vec{a}$ & $\begin{array}{l}\hat{\sigma} \\
\infty\end{array}$ & $\begin{array}{l}0 \\
0 \\
\infty\end{array}$ & $\frac{1}{6}$ & 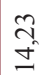 & $\begin{array}{l}n \\
2 \\
2\end{array}$ & $\begin{array}{l}\text { ๙ू } \\
\text { ปे }\end{array}$ & $\stackrel{2}{\check{2}}$ & \\
\hline & & & 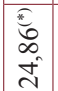 & $\begin{array}{l}\infty \\
\vec{\omega} \\
\sim\end{array}$ & $\begin{array}{l}\vec{\alpha} \\
\hat{m}\end{array}$ & $\begin{array}{l}\text { बे } \\
\text { ते }\end{array}$ & 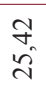 & $\begin{array}{l}\hat{a} \\
\hat{i}\end{array}$ & 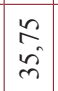 & ঐे. & $\begin{array}{l}\infty \\
\infty \\
\infty \\
\sim\end{array}$ & $\begin{array}{l}\hat{\sigma} \\
\text { of } \\
\text { in }\end{array}$ & $\begin{array}{l}8 \\
\text { in }\end{array}$ & $\begin{array}{l}m \\
m \\
\hat{n}\end{array}$ & $\begin{array}{l}\hat{n} \\
\hat{n} \\
\text { na }\end{array}$ & $\begin{array}{l}n \\
n \\
\infty\end{array}$ & $\frac{ \pm}{\lambda^{\prime}}$ & $\begin{array}{l}\stackrel{n}{m} \\
\stackrel{f}{d}\end{array}$ & $\begin{array}{l}\tilde{J} \\
\dot{\tau} \\
\end{array}$ & $\begin{array}{l}\stackrel{\circ}{a} \\
\vec{i}\end{array}$ & $\begin{array}{l}\infty \\
\infty \\
\infty \\
\infty\end{array}$ & 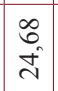 & $\begin{array}{l}8 \\
\stackrel{-}{=}\end{array}$ & 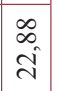 & \\
\hline & & & $\vec{\Xi}$ & $\begin{array}{l}\stackrel{\text { }}{ } \\
\text { त̂ }\end{array}$ & $\begin{array}{l}\text { तે } \\
\text { }\end{array}$ & $\frac{0}{\sqrt{n}}$ & $\begin{array}{l}\stackrel{+}{N} \\
\underset{\sim}{v}\end{array}$ & $\begin{array}{l}8 \\
\stackrel{8}{0} \\
\text { in }\end{array}$ & $\begin{array}{l} \pm \\
0 \\
0 \\
0\end{array}$ & $\begin{array}{l}\stackrel{n}{a} \\
\stackrel{+}{d}\end{array}$ & $\begin{array}{l}\hat{\hat{\sigma}} \\
\hat{\Omega}\end{array}$ & 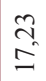 & $\begin{array}{l}0 \\
\text { m. } \\
\tilde{n}\end{array}$ & $\begin{array}{l}\stackrel{0}{ } \\
\vec{i}\end{array}$ & $\begin{array}{l}\hat{n} \\
\infty \\
\infty\end{array}$ & $\begin{array}{l}\overrightarrow{0} \\
\stackrel{0}{0}\end{array}$ & $\begin{array}{l}\vec{\infty} \\
\vec{\sim}\end{array}$ & $\begin{array}{l}\tilde{n} \\
\tilde{n}\end{array}$ & $\begin{array}{l}\stackrel{+}{2} \\
\text { ते }\end{array}$ & $\begin{array}{l}\stackrel{m}{\sim} \\
\ddot{\sim}\end{array}$ & $\begin{array}{l}n \\
\infty \\
\infty \\
\infty\end{array}$ & $\begin{array}{l}\stackrel{2}{\cong} \\
=\end{array}$ & $\begin{array}{l}\bar{n} \\
\tilde{n}\end{array}$ & $\begin{array}{l}n \\
\hat{0} \\
-1\end{array}$ & \\
\hline & & $\begin{array}{l}\text { ڤ̆ } \\
\text { हี }\end{array}$ & $\begin{array}{l}n \\
n \\
n\end{array}$ & 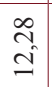 & $\stackrel{\infty}{\stackrel{\infty}{\simeq}}$ & 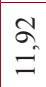 & $\begin{array}{l}3 \\
0 \\
0\end{array}$ & $\begin{array}{l}\vec{\sim} \\
n\end{array}$ & $\begin{array}{l}m \\
\hat{0} \\
0\end{array}$ & $\begin{array}{l}0 \\
\pm \\
\pm\end{array}$ & $\stackrel{\partial}{\varrho}$ & $\stackrel{ }{\stackrel{2}{i}}$ & $\begin{array}{l}\stackrel{g}{\sigma} \\
\stackrel{g}{2}\end{array}$ & $\stackrel{8}{=}$ & $\begin{array}{l}n \\
a \\
a\end{array}$ & $\stackrel{0}{\varrho}$ & $\stackrel{2}{\Leftrightarrow}$ & $\stackrel{+}{\stackrel{ \pm}{\simeq}}$ & $\begin{array}{l}0 \\
0 \\
\simeq\end{array}$ & $\begin{array}{l}m \\
\pm \\
\pm\end{array}$ & $\begin{array}{l}\infty \\
\infty \\
\infty\end{array}$ & $\underset{+}{\mathscr{\infty}}$ & $\stackrel{n}{\approx}$ & $\underset{f}{\stackrel{f}{r}}$ & \\
\hline & & & $\begin{array}{l}\frac{\pi}{4} \\
6 \\
\stackrel{0}{0}\end{array}$ & $\begin{array}{l}\stackrel{\searrow}{2} \\
\text { I }\end{array}$ & à & $\begin{array}{l}\tilde{\sigma} \\
\Xi\end{array}$ & $\stackrel{m}{\infty}$ & $\begin{array}{l}8 \\
\stackrel{0}{0} \\
\therefore\end{array}$ & $\begin{array}{l}\infty \\
\sim \\
\sim\end{array}$ & $\vec{m}$ & $\begin{array}{l}\infty \\
\hat{n} \\
\Omega\end{array}$ & $\begin{array}{l}n \\
\text { in } \\
\text { in }\end{array}$ & $\begin{array}{l}\stackrel{9}{+} \\
\infty \\
\infty\end{array}$ & $\stackrel{2}{6}$ & $\begin{array}{c}\tilde{\sigma} \\
\tilde{\Omega}\end{array}$ & $\begin{array}{l}n \\
0 \\
n\end{array}$ & $\begin{array}{l}\tilde{\infty} \\
\hat{\sigma}_{0}\end{array}$ & aे & $\begin{array}{l}2 \\
2 \\
=\end{array}$ & $\begin{array}{l}\infty \\
\stackrel{2}{2}\end{array}$ & $\begin{array}{l}\infty \\
\infty \\
2 \\
-2\end{array}$ & $\begin{array}{l}\partial \\
\hat{2}\end{array}$ & $\frac{m}{\infty}$ & $\begin{array}{l}\text { ô } \\
\text { I }\end{array}$ & \\
\hline 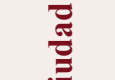 & & & $\begin{array}{l}\overrightarrow{\hat{~}} \\
\vec{\sim}\end{array}$ & $\begin{array}{l}n \\
\hat{n} \\
\tilde{v}\end{array}$ & $\begin{array}{l}\text { î } \\
\text { ते }\end{array}$ & 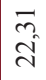 & 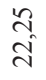 & $\begin{array}{l}\overrightarrow{0} \\
\tilde{d}\end{array}$ & 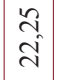 & $\begin{array}{l}\tilde{i} \\
\tilde{v}\end{array}$ & $\frac{n}{\vec{v}}$ & $\begin{array}{l}\vec{\sim} \\
\text { v }\end{array}$ & 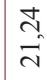 & $\begin{array}{l}\bar{b} \\
\vec{i}\end{array}$ & $\begin{array}{l}\tilde{N} \\
\tilde{N}\end{array}$ & $\begin{array}{l}\vec{b} \\
\vec{i}\end{array}$ & $\frac{8}{i}$ & 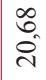 & 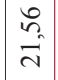 & $\begin{array}{l}= \\
\overrightarrow{\vec{v}}\end{array}$ & $\begin{array}{l}\circ \\
\stackrel{\text { సे }}{\text { s. }}\end{array}$ & $\begin{array}{l} \pm \\
\vec{i}\end{array}$ & 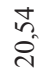 & $\overrightarrow{\vec{\sigma}}$ & \\
\hline 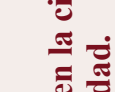 & 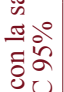 & 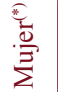 & $\frac{8}{\vec{i}}$ & $\frac{\tilde{v}}{\vec{n}}$ & $\begin{array}{l}m \\
\hat{i} \\
\text { in }\end{array}$ & $\stackrel{\circ}{\stackrel{i}{i}}$ & $\frac{\tilde{i}}{i}$ & $\begin{array}{l}\infty \\
\ddot{i} \\
\stackrel{i}{i}\end{array}$ & $\frac{\tilde{\theta}}{\hat{\lambda}}$ & $\begin{array}{l}\vec{\sigma} \\
\vec{i}\end{array}$ & $\begin{array}{l}\infty \\
\infty \\
\stackrel{N}{*}\end{array}$ & $\begin{array}{l}\text { ڤू } \\
\text { ஸे }\end{array}$ & $\begin{array}{l}\infty \\
\stackrel{0}{0}\end{array}$ & $\begin{array}{l}\text { fे } \\
\text { ¿े }\end{array}$ & $\begin{array}{l}\stackrel{8}{\vec{\lambda}} \\
\vec{\lambda}\end{array}$ & 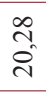 & $\hat{\imath}$ & $\begin{array}{l}n \\
2 \\
\Omega\end{array}$ & $\begin{array}{l}0 \\
\text { ñ. } \\
\text { ते }\end{array}$ & $\begin{array}{l}\stackrel{2}{2} \\
\stackrel{2}{2}\end{array}$ & $\begin{array}{l}3 \\
0 \\
2\end{array}$ & $\begin{array}{l}+ \\
\stackrel{2}{2}\end{array}$ & $\begin{array}{l}\stackrel{\infty}{N} \\
\stackrel{2}{2}\end{array}$ & 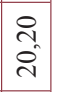 & \\
\hline 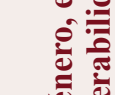 & : & & 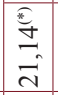 & 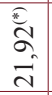 & $\begin{array}{l}n \\
\text { i } \\
\text { in }\end{array}$ & $\frac{\vec{b}}{\vec{i}}$ & $\begin{array}{l}\overrightarrow{0} \\
\vec{d}\end{array}$ & $\begin{array}{l}\tilde{m} \\
\vec{\sim}\end{array}$ & $\begin{array}{l}\vec{b} \\
\vec{i}\end{array}$ & $\begin{array}{l}\text { S. } \\
\vec{i}\end{array}$ & $\begin{array}{l}\infty \\
m \\
\vec{\sim}\end{array}$ & $\stackrel{\infty}{n}$ & $\begin{array}{l}\text { in } \\
\text { in }\end{array}$ & $\begin{array}{l}\text { d } \\
\text { ì }\end{array}$ & $\begin{array}{l}\overrightarrow{\dot{O}} \\
\overrightarrow{\mathrm{d}}\end{array}$ & 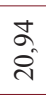 & $\begin{array}{l}\vec{f} \\
\stackrel{\Omega}{\hat{~}}\end{array}$ & $\begin{array}{l}\text { oे } \\
\text { iे }\end{array}$ & $\begin{array}{l}\tilde{\gamma} \\
\hat{\tilde{\nu}}\end{array}$ & $\begin{array}{l}\stackrel{n}{n} \\
\stackrel{2}{n}\end{array}$ & ते & $\begin{array}{l}\hat{t} \\
\hat{i}\end{array}$ & 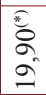 & $\begin{array}{l}\bar{\infty} \\
\text { ते }\end{array}$ & \\
\hline 更 & 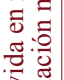 & & $\begin{array}{l}\infty \\
\overrightarrow{2}\end{array}$ & $\frac{ \pm}{8}$ & $\begin{array}{l}\text { ¿े } \\
\text { ते }\end{array}$ & 옹 & $\begin{array}{l}\text { J్ } \\
\stackrel{2}{2}\end{array}$ & $\begin{array}{l}0 \\
\text { ñ. } \\
\stackrel{\sim}{1}\end{array}$ & 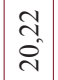 & $\stackrel{q}{q}$ & $\begin{array}{l}2 \\
\infty \\
0\end{array}$ & $\frac{n}{2}$ & $\begin{array}{l}\infty \\
0\end{array}$ & $\begin{array}{l}\infty \\
\infty \\
2\end{array}$ & $\begin{array}{l}\infty \\
0 \\
0 \\
2\end{array}$ & ลू. & थै & $\begin{array}{l}\text { ర్ } \\
\text { @- }\end{array}$ & $\begin{array}{l}\vec{\infty} \\
\stackrel{2}{2}\end{array}$ & $\frac{m}{a}$ & $\begin{array}{l}\tilde{2} \\
2\end{array}$ & $\begin{array}{l}\tilde{\sigma} \\
\sigma \\
\Omega\end{array}$ & $\begin{array}{l}0 \\
n \\
2 \\
2\end{array}$ & $\begin{array}{l}n \\
\stackrel{n}{2} \\
\stackrel{2}{2}\end{array}$ & \\
\hline 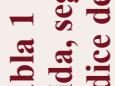 & 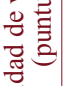 & 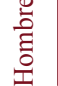 & $\begin{array}{l}\vec{\sigma} \\
\stackrel{\infty}{\infty}\end{array}$ & $\begin{array}{l}\infty \\
\infty \\
\infty\end{array}$ & $\begin{array}{l}0 \\
0 \\
\infty\end{array}$ & $\begin{array}{l}2 \\
\infty \\
\infty\end{array}$ & 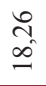 & $\begin{array}{l}\infty \\
\infty \\
\infty\end{array}$ & $\begin{array}{l}\bar{\sigma} \\
\infty \\
\sigma\end{array}$ & $\frac{n}{\infty}$ & $\begin{array}{l}6 \\
0 \\
0 \\
0\end{array}$ & \begin{tabular}{l}
\multirow{2}{*}{} \\
$\infty$ \\
-
\end{tabular} & $\begin{array}{l}n \\
n \\
\infty\end{array}$ & $\begin{array}{l}n \\
\infty \\
\infty \\
\infty\end{array}$ & $\begin{array}{l}\stackrel{2}{=} \\
=\end{array}$ & $\begin{array}{l}\stackrel{m}{+} \\
\infty \\
\infty\end{array}$ & 吉 & $\begin{array}{l}m \\
\text { on } \\
\infty\end{array}$ & $\begin{array}{l}\stackrel{m}{+} \\
\infty \\
\mathscr{0}\end{array}$ & $\begin{array}{l}\vec{m} \\
\infty\end{array}$ & g. & 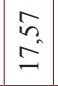 & $\begin{array}{l}\Omega \\
\Omega\end{array}$ & $\begin{array}{l}0 \\
0 \\
\infty\end{array}$ & \\
\hline F & & & 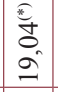 & $\begin{array}{l}\tilde{f} \\
\stackrel{2}{2}\end{array}$ & $\stackrel{m}{2}$ & $\begin{array}{l}\stackrel{+}{a} \\
\text { a }\end{array}$ & $\begin{array}{l} \pm \\
\infty \\
\infty\end{array}$ & $\begin{array}{l}\infty \\
2 \\
2\end{array}$ & $\begin{array}{l}n \\
2 \\
2\end{array}$ & $\begin{array}{l}\stackrel{\infty}{\sim} \\
\infty \\
\infty\end{array}$ & $\begin{array}{l}\hat{2} \\
\hat{2}\end{array}$ & $\begin{array}{l}2 \\
\infty \\
\infty\end{array}$ & กิ & $\begin{array}{l}\overrightarrow{2} \\
\hat{2}\end{array}$ & $\begin{array}{l}J_{0} \\
\infty \\
\infty\end{array}$ & $\frac{1}{9}$ & $\begin{array}{l}3 \\
\infty \\
\infty\end{array}$ & $\hat{\alpha}$ & $\stackrel{\Xi}{a}$ & $\begin{array}{l}0 \\
0 \\
\sigma\end{array}$ & $\sqrt{-}$ & $\begin{array}{l}0 \\
0 \\
\infty \\
\infty \\
-1\end{array}$ & $\begin{array}{l}\mathbb{J} \\
\infty \\
-\end{array}$ & $\frac{7}{\infty}$ & \\
\hline 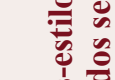 & & & $\begin{array}{l}\stackrel{\infty}{2} \\
\text { ô }\end{array}$ & $\begin{array}{l}8 \\
6 \\
6\end{array}$ & तु & $\begin{array}{l}\infty \\
\infty \\
\tilde{b}\end{array}$ & $\begin{array}{l}3 \\
\hat{6}\end{array}$ & $\frac{8}{\pi}$ & $\mid \begin{array}{l}\infty \\
\infty \\
\infty \\
0\end{array}$ & $\stackrel{\tilde{n}}{i}$ & $\begin{array}{l}\hat{2} \\
i\end{array}$ & $\begin{array}{l}0 \\
\hat{\sigma} \\
0\end{array}$ & $\begin{array}{l}0 \\
\infty \\
\stackrel{1}{1}\end{array}$ & $\begin{array}{c}\text { a } \\
\text { is }\end{array}$ & 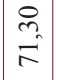 & $\begin{array}{l}z \\
i \\
i\end{array}$ & ते & $\begin{array}{l}2 \\
\hat{\delta} \\
\infty\end{array}$ & $\begin{array}{l}7 \\
i \\
i\end{array}$ & $\begin{array}{l}\hat{m} \\
\infty \\
\infty\end{array}$ & 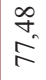 & $\begin{array}{l}\bar{\sigma} \\
\hat{\sigma}\end{array}$ & $\begin{array}{l}\text { t } \\
\text { i } \\
\infty\end{array}$ & तू & \\
\hline 을 를 즐 & 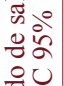 & $\stackrel{\stackrel{\pi}{c}}{\stackrel{0}{3}}$ & $\begin{array}{l}\stackrel{2}{\hat{8}} \\
8\end{array}$ & $\begin{array}{l}\hat{n} \\
\text { n̊. } \\
\text { ñ }\end{array}$ & $\begin{array}{l}\tilde{0} \\
\ddot{n}\end{array}$ & $\begin{array}{l}\infty \\
\infty \\
\text { in }\end{array}$ & $\hat{\hat{n}}$ & $\begin{array}{l}\infty \\
0 \\
0 \\
0\end{array}$ & $\begin{array}{l}3 \\
6 \\
6 \\
n\end{array}$ & $\begin{array}{l}0 \\
\infty \\
i n\end{array}$ & $\mid \begin{array}{l}0 \\
0 \\
8 \\
8\end{array}$ & $\begin{array}{l}0 \\
n \\
n \\
n\end{array}$ & đु & $\begin{array}{l}n \\
n \\
8 \\
8\end{array}$ & $\begin{array}{l}\stackrel{R}{n} \\
\stackrel{n}{n}\end{array}$ & $\begin{array}{l}8 \\
8 \\
8\end{array}$ & $\frac{6}{6}$ & o. & $\begin{array}{l}\text { ڤे } \\
\text { ले }\end{array}$ & 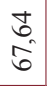 & $\begin{array}{l}+ \\
\infty \\
0 \\
0\end{array}$ & $\begin{array}{c}2 \\
0 \\
6\end{array}$ & $\stackrel{8}{-}$ & $\begin{array}{l}\sqrt{0} \\
0 \\
0 \\
0\end{array}$ & \\
\hline$\vec{D}^{\circ}$ & 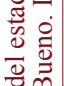 & & $\begin{array}{l}\frac{\pi}{0} \\
0 \\
\infty \\
0 \\
0\end{array}$ & 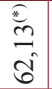 & $\frac{n}{6}$ & $\begin{array}{l}\infty \\
\infty \\
0 \\
n\end{array}$ & $\begin{array}{l}\infty \\
-1\end{array}$ & $\begin{array}{l}2 \\
\hat{b}\end{array}$ & $\begin{array}{l}0 \\
\tilde{a} \\
\tilde{b}\end{array}$ & $\begin{array}{l}\text { If } \\
\text { ti }\end{array}$ & $\mid \begin{array}{l}-\infty \\
\delta^{n}\end{array}$ & $\frac{9}{6}$ & $\begin{array}{l}n \\
\text { in } \\
\text { in }\end{array}$ & \begin{tabular}{l}
$\infty$ \\
\multirow{1}{1}{} \\
$\delta$ \\
$\delta$
\end{tabular} & $\begin{array}{l}0 \\
n \\
n \\
n\end{array}$ & $\begin{array}{l}n \\
\hat{n} \\
\text { so }\end{array}$ & 守 & $\begin{array}{l}n \\
n \\
n\end{array}$ & $\begin{array}{l}\hat{6} \\
8 \\
0\end{array}$ & $\begin{array}{l}8 \\
i \\
i\end{array}$ & $\frac{1}{a}$ & $\vec{m}$ & $\begin{array}{l}\infty \\
\infty \\
0 \\
0\end{array}$ & 慗 & \\
\hline en & 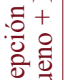 & & $\begin{array}{l}3 \\
\infty \\
\infty \\
\infty\end{array}$ & $\begin{array}{l}\infty \\
\infty \\
\infty \\
\infty\end{array}$ & $\begin{array}{l}\hat{n} \\
\infty \\
\infty\end{array}$ & $\begin{array}{l}\infty \\
\infty \\
\stackrel{1}{\infty}\end{array}$ & $\stackrel{f}{\infty}$ & $\begin{array}{l}\tilde{n} \\
\tilde{\infty} \\
\infty\end{array}$ & $\frac{n}{\infty}$ & $\begin{array}{l}8 \\
0 \\
\infty\end{array}$ & $\mid \begin{array}{c}8 \\
\tilde{\infty} \\
\infty\end{array}$ & $\begin{array}{l}\approx \\
\approx \\
n\end{array}$ & $\stackrel{\infty}{\stackrel{\infty}{i f}}$ & $\frac{t}{\infty}$ & $\begin{array}{l}n \\
\hat{\infty} \\
\infty\end{array}$ & $\begin{array}{l}n \\
\tilde{5} \\
+\end{array}$ & $\begin{array}{l}\infty \\
\curvearrowright \\
\text { त̂ }\end{array}$ & $\begin{array}{l}\stackrel{ \pm}{d} \\
\stackrel{\infty}{0}\end{array}$ & $\begin{array}{l}\bar{\sigma} \\
\tilde{\infty}\end{array}$ & $\begin{array}{l}0 \\
2 \\
\infty \\
\infty\end{array}$ & $\frac{m}{\hat{\infty}}$ & $\begin{array}{l} \pm \\
\dot{\infty}\end{array}$ & बे & \begin{tabular}{l}
$\infty$ \\
\multirow{1}{*}{} \\
$\infty$
\end{tabular} & \\
\hline हूँ & 这焉 & है & $\begin{array}{l}0 \\
\stackrel{0}{0}\end{array}$ & $\begin{array}{l}2 \\
2 \\
8 \\
8\end{array}$ & $\begin{array}{l}8 \\
\text { : }\end{array}$ & $\begin{array}{l}\hat{6} \\
\tilde{6}\end{array}$ & $\frac{m}{2}$ & $\begin{array}{l}\stackrel{m}{0} \\
\infty \\
\infty\end{array}$ & $\stackrel{f}{\tilde{F}}$ & $\begin{array}{l}\hat{m} \\
n \\
n\end{array}$ & $\begin{array}{l}\hat{n} \\
\hat{n}\end{array}$ & $\stackrel{\infty}{\stackrel{\sigma}{\sigma}}$ & $\begin{array}{l}\text { N } \\
\text { ה }\end{array}$ & $\begin{array}{l}\text { กิ } \\
\text { อิ }\end{array}$ & 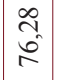 & $\begin{array}{l}\stackrel{n}{m} \\
\stackrel{n}{n}\end{array}$ & $\stackrel{\infty}{\infty}$ & $\begin{array}{l}\text { to } \\
\stackrel{\sim}{n}\end{array}$ & $\begin{array}{l}n \\
n \\
\tilde{n}\end{array}$ & $\vec{i}$ & $\frac{R}{i}$ & $\begin{array}{l}0 \\
0 \\
2 \\
2\end{array}$ & $\begin{array}{l}\text { P } \\
\text { i }\end{array}$ & $\begin{array}{l}\hat{m} \\
\hat{n}\end{array}$ & \\
\hline$\frac{1}{2}$ & $a^{\circ}$ & & 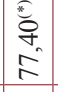 & $\begin{array}{l}\text { î } \\
\text { i }\end{array}$ & 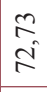 & $\stackrel{ }{i}$ & $\begin{array}{l}\infty \\
\infty \\
i n\end{array}$ & \begin{tabular}{l}
$\stackrel{⿱}{n}$ \\
\multirow{f}{*}{}
\end{tabular} & $\begin{array}{l}2 \\
\hat{0}\end{array}$ & $\begin{array}{l}n \\
\hat{n} \\
\infty\end{array}$ & $\begin{array}{l}\hat{n} \\
\hat{i}\end{array}$ & $\begin{array}{l}\tilde{\delta} \\
\infty \\
0 \\
0\end{array}$ & $\begin{array}{l}\stackrel{n}{n} \\
\stackrel{2}{n}\end{array}$ & $\frac{2}{2}$ & $\begin{array}{c}n \\
\infty \\
\infty\end{array}$ & $\begin{array}{l}8 \\
2 \\
2\end{array}$ & 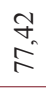 & $\begin{array}{l}\vec{J} \\
\infty \\
\infty\end{array}$ & $\begin{array}{l}\text { त̃ } \\
\infty \\
\infty\end{array}$ & $\begin{array}{l}n \\
\infty \\
\infty \\
\infty\end{array}$ & $\frac{i}{\stackrel{2}{2}}$ & $\mid \begin{array}{l}\infty \\
0 \\
\dot{+} \\
\dot{\infty}\end{array}$ & $\stackrel{5}{\infty}$ & $\begin{array}{l}\infty \\
0 \\
0 \\
2\end{array}$ & $\overrightarrow{\vec{b}_{0}}$ \\
\hline$\frac{\sqrt[\pi]{\frac{\pi}{0}}}{\frac{0}{\tilde{T}}}$ & & & 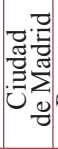 & & 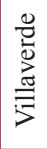 & 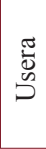 & 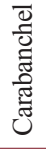 & . & 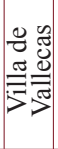 & 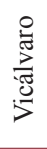 & 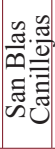 & & 这 & $\begin{array}{l}\frac{N}{\pi} \\
\frac{\pi}{\pi} \\
\frac{\pi}{0} \\
\Sigma\end{array}$ & 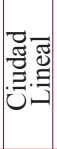 & 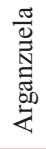 & 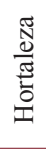 & 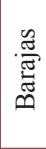 & 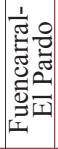 & 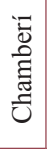 & 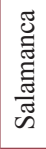 & 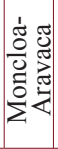 & 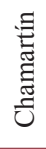 & : 을 & 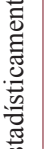 \\
\hline & & & & - & $\sim$ & $m$ & $\nabla$ & in & 0 & $r$ & $\infty$ & $a$ & 으 & $=$ & $\simeq$ & $\stackrel{m}{=}$ & \pm & $\cong$ & $\stackrel{0}{1}$ & I & $\infty$ & 2 & ๙ิ & $\vec{\sim}$ & \\
\hline
\end{tabular}




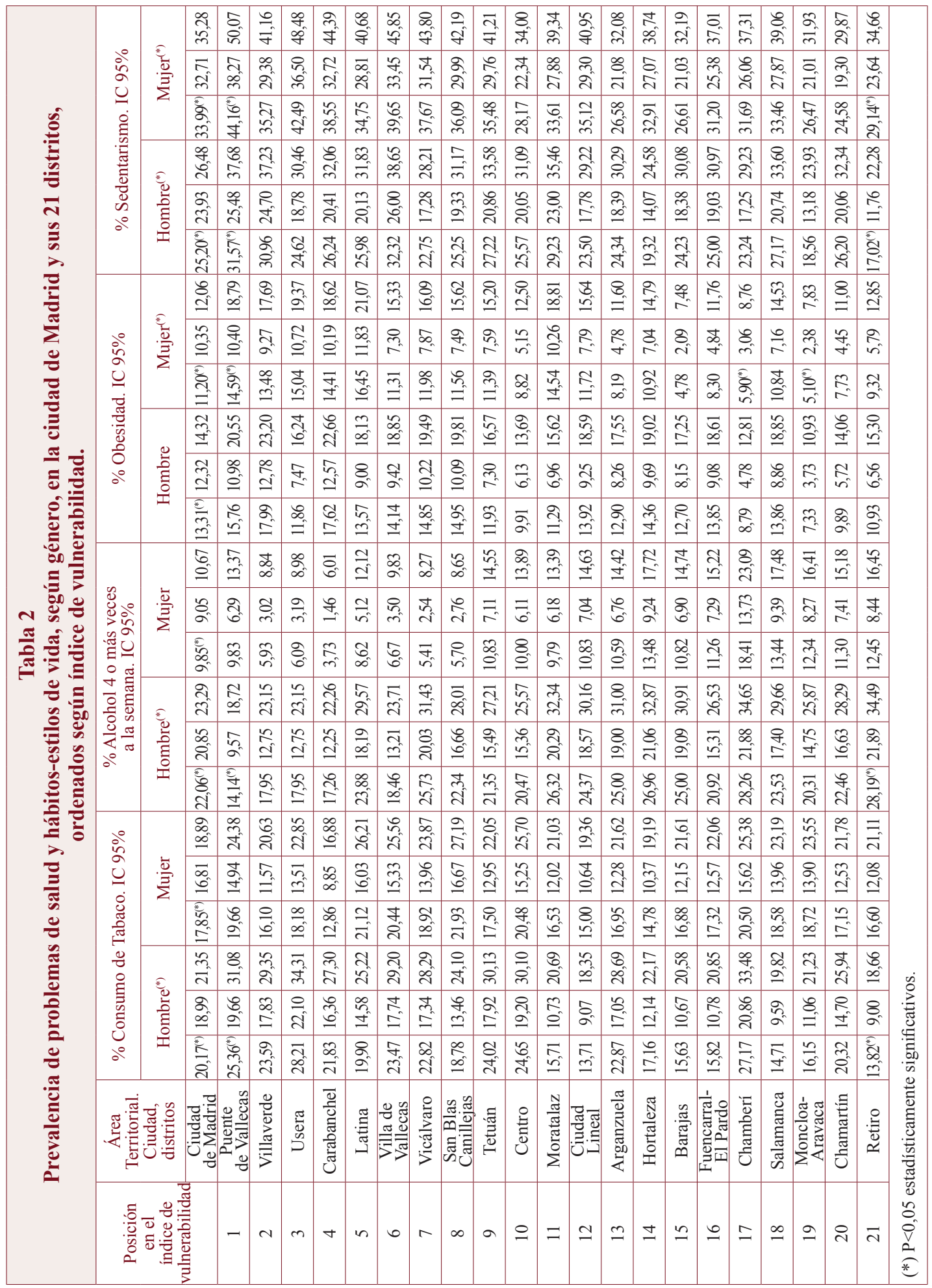


A través de los mapas de la figura 1 se visualiza la distribución de la vulnerabilidad por distritos, según el Indicador de vulnerabilidad-ranking elaborado en 2017, así como la concentración de los problemas de autopercepción del estado de salud, mayor en mujeres, y con similar superposición en el territorio más vulnerable, como en CVRS y en salud mental. En el caso

\section{Figura 1}

Vulnerabilidad, autopercepción del estado de salud, calidad de vida en relación con la salud y salud mental, consumo de tabaco y alcohol, obesidad y sedentarismo.

Distribución territorial por los distritos de la ciudad de Madrid. Hombres y mujeres.

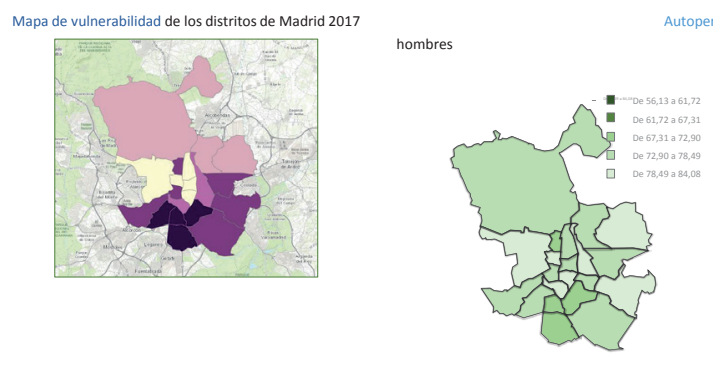

stado de salud referido por la población de los

distritos de Madrid 2017

mujeres

Consumo de tabaco referido por la población de los distritos de Madrid 2017
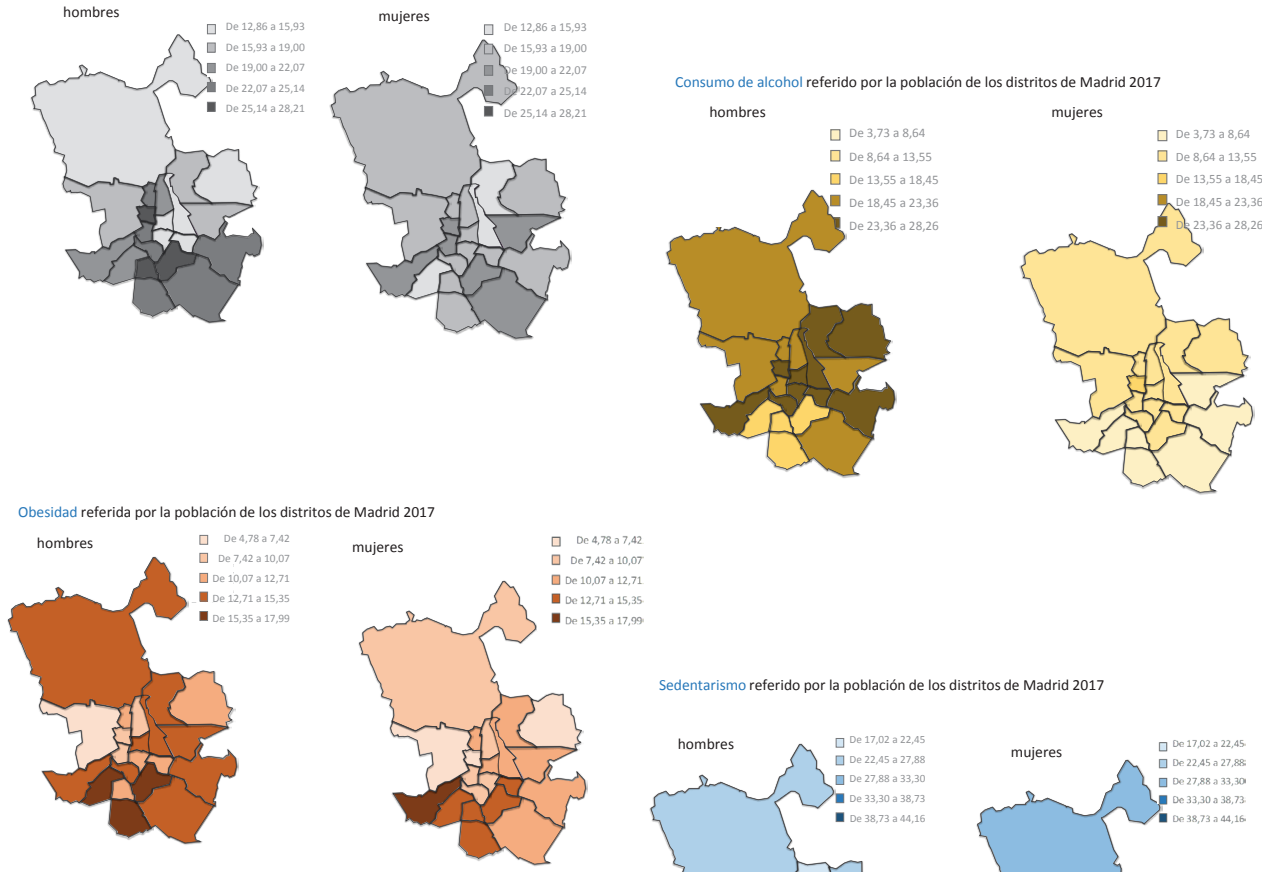

Sedentarismo referido por la población de los distritos de Madrid 2017
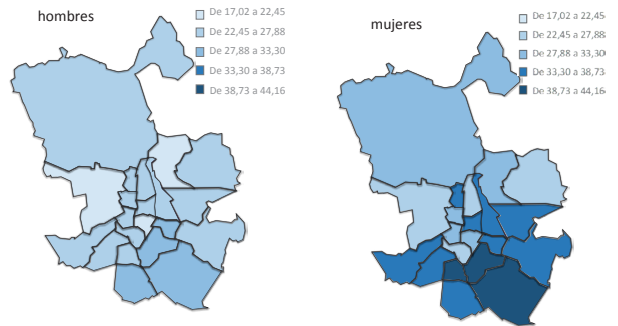
del consumo de tabaco, hubo mayor prevalencia en hombres, también con un patrón territorial similar al de la vulnerabilidad. No ocurrió así con el alcohol en varones, con un patrón más centralista en cuanto al territorio. Por último, en obesidad y en sedentarismo fue igualmente superponible el patrón de territorio más vulnerable en las mujeres.

En la tabla 3 se presentan los índices de correlación y su significación entre el indicador de vulnerabilidad y los problemas de salud-estilos de vida, desagregados por sexo. Los niveles de asociación fueron muy altos, con $\mathrm{p}<0,01$ para la vulnerabilidad y calidad de vida en relación con la salud, y la percepción del estado de salud (en ambos sexos) y la posibilidad de tener una enfermedad mental en las mujeres. Igualmente, fue significativa la relación en el consumo de tabaco en hombres, y de alcohol en ambos sexos, si bien en este último caso de forma inversa. Para la obesidad y el sedentarismo también fue significativa la asociación tanto en hombres como en mujeres.

En la figura 2 se muestran las rectas de regresión lineal entre vulnerabilidad y problemas de salud, explicando con modelos de regresión el porcentaje que la desviación de la varianza expresa por la vulnerabilidad en los problemas de salud, mediante el indicador de asociación $\mathrm{R}^{2}$. Nos encontramos con que los datos más relevantes correspondieron a la calidad de vida en relación con la salud en mujeres. También, la autopercepción del estado de salud, así como el sedentarismo y la obesidad, también en mujeres, con valores que supusieron entre el 60\% y el $80 \%$ de la varianza explicada. Fueron, por orden de valores: CVRS, con un $\mathrm{R}^{2}$ de 0,71 ; autopercepción del estado de salud (bueno + muy

\begin{tabular}{|c|c|c|c|c|}
\hline \multicolumn{5}{|c|}{$\begin{array}{l}\text { Tabla } 3 \\
\text { Índices de correlación y su significación entre las variables de salud e indicador } \\
\text { de vulnerabilidad } 2017 \text { para los } 21 \text { distritos de la ciudad de Madrid, hombres y mujeres. }\end{array}$} \\
\hline \multicolumn{5}{|c|}{ Indice de vulnerabilidad 2017} \\
\hline \multirow{2}{*}{ Variables } & $\begin{array}{l}\text { Correlación } \\
\text { de Pearson }\end{array}$ & \multirow{2}{*}{ Sig. } & $\begin{array}{l}\text { Correlación } \\
\text { de Pearson }\end{array}$ & \multirow{2}{*}{ Sig. } \\
\hline & Hombres & & Mujeres & \\
\hline $\begin{array}{c}\text { Autopercepción } \\
\text { del estado de salud (Muy bueno+Bueno) }\end{array}$ & $-0,656^{(* *)}$ & 0,001 & $-0,838^{(* *)}$ & 0,0001 \\
\hline $\begin{array}{c}\text { Calidad Vida en relaccion } \\
\text { con la salud }\end{array}$ & $0,653^{(* *)}$ & 0,001 & $0,840^{(* *)}$ & 0,0001 \\
\hline Salud mental-GHQ12 & 0,30 & 0,193 & $0,641^{(* *)}$ & 0,002 \\
\hline Consumo de Tabaco & $0,573^{(* *)}$ & 0,007 & 0,09 & 0,709 \\
\hline $\begin{array}{c}\text { Alcohol } 4 \text { o más } \\
\text { veces a la semana }\end{array}$ & $-0,712^{(* *)}$ & 0,0001 & $-0,724^{(* *)}$ & 0,0001 \\
\hline Obesidad & $0,637^{(* *)}$ & 0,002 & $0,775^{(* *)}$ & 0,0001 \\
\hline Sedentarismo & $0,607^{(* *)}$ & 0,003 & $0,828^{(* *)}$ & 0,0001 \\
\hline \multicolumn{5}{|c|}{$\begin{array}{l}\text { (*) La correlación es significativa al nivel } 0,05 \text { (bilateral). }(* *) \text { La correlación es sigificativa al nivel } \\
0,01 \text { (bilateral). }\end{array}$} \\
\hline
\end{tabular}




\section{Figura 2}

Líneas de regresión entre la prevalencia de problemas de salud en los 21 distritos de la ciudad de Madrid y la vulnerabilidad (IVR) hombres y mujeres.

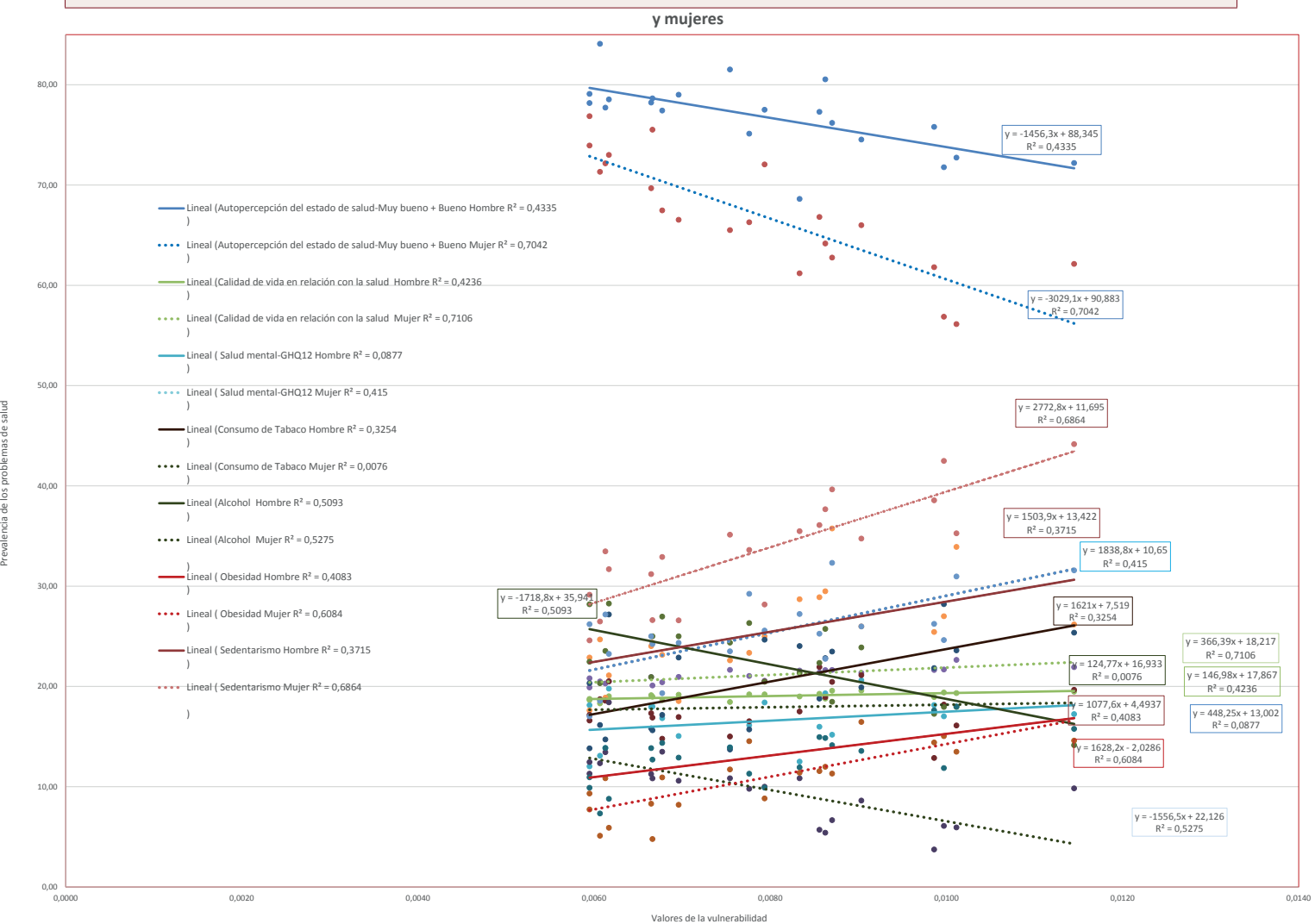

bueno), 0,70; sedentarismo, 0,68 ; obesidad, 0,60 . La salud mental-GHQ-12, marcó 0,41 en mujeres; el alcohol, 0,50, tanto en hombres como en mujeres; y el consumo de tabaco, 0,32 en hombres, lo que explicó entre el 30 y el $60 \%$ de la varianza.

En el figura 3 se ordenan las líneas de regresión de las variables de salud, según vinculación a la vulnerabilidad, a través de su derivada. De arriba-abajo se representan las líneas por la proximidad a la vulnerabilidad. Secuenciadas en el orden del valor, fueron las siguientes: 1-Autopercepción del estado de salud en mujeres, 2-Sedentarismo en mujeres, 3-Salud mental en mujeres, 4-Alcohol en hombres, 5-Sobrepeso en mujeres, 6-Tabaco en hombres, 7-Alcohol en mujeres, 8-Sedentarismo en hombres, 9-Autopercepción del estado de salud en hombres, 10-Sobrepeso en hombres, 11-Salud mental en hombres, 12-CVRS en mujeres, 13 CVRS en hombres, 14-Tabaco en mujeres. 


\section{Figura 3}

Aproximación al eje de vulnerabilidad de las líneas de salud y los hábitos-estilos de vida a través de sus derivadas.

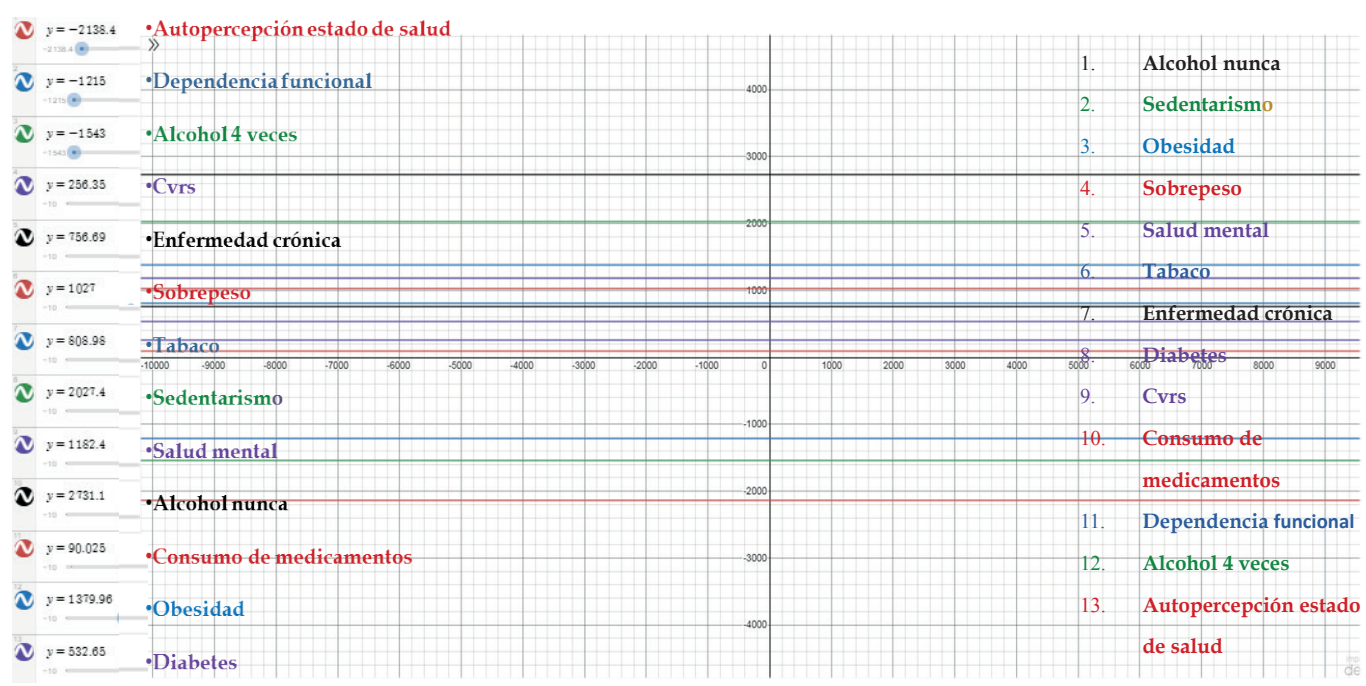

\section{DISCUSIÓN}

Los indicadores de autopercepción del estado de salud y CVRS presentan diferencias muy significativas desde el punto de vista territorial, en cuanto a prevalencia para el indicador en conjunto, es decir, teniendo en cuenta ambos sexos. La significación en la diferencia también es entre ambos sexos. Este matiz de género se permeabiliza claramente hacia las mujeres, reflejándose tanto en la autopercepción del estado de salud como en la CVRS la diferencia de los indicadores de en los distritos más y menos vulnerables. La varianza explicada para ambos por este concepto está entre el $60-80 \%$, si bien en las mujeres se explica en casi el $85 \%$ en ambos casos. La aproximación por las líneas de regresión es alta, con $\mathrm{R}^{2} \mathrm{de}$ 0,70 para los dos indicadores. Estos resultados se corroboran con otros estudios que vinculan claramente la salud con la vulnerabilidad y la desigualdad social, y que tienen gran relación territorial, representando a las mujeres como el grupo más afectado en este caso. Se apunta claramente a la diferencia entre distritos para los problemas de salud en las mujeres ${ }^{(23,24,28)}$.

En cuanto a la obesidad y el sedentarismo, se encuentran diferencias importantes en cuanto a la presencia territorial para el conjunto del indicador, siendo significativa la diferencia por género, y correspondiendo a las mujeres la explicación más marcada en los territorios extremos por obesidad. En el caso del sedentarismo, la diferencia se refleja entre un territorio más y menos vulnerable, tanto para hombres como para mujeres. El nivel de correlación es alto, pero en el caso de las mujeres la varianza explicada por la vulnerabilidad, tanto para el sedentarismo como la obesidad, está en torno al $80 \%$. Los valores de $\mathrm{R}^{2}$ para mujeres superan el 0,6. Diferentes estudios vinculan la clase social y la desigualdad con los problemas de obesidad, el sedentarismo y el sobrepeso. En este caso, es 
en las mujeres igualmente dónde los patrones están más definidos ${ }^{(9,10,23)}$.

El alcohol se presenta como un área muy significativa en cuanto a prevalencia en territorios más y menos vulnerables para el conjunto del indicador. La diferencia es significativa para el sexo, y es en los hombres donde las diferencias territoriales son acusadas entre los distritos más y menos vulnerables, si bien en este caso el comportamiento es inverso, es decir, más consumo en zonas menos vulnerables y menos en las más. El consumo de alcohol está claramente relacionado de forma inversa cuando lo asociamos con los distritos y la vulnerabilidad. Tanto por sus vínculos territoriales en IC 95\%, como por el patrón de consumo, es en las áreas más vulnerables dónde se consume menos y en los menos vulnerables dónde más. Este comportamiento concuerda claramente con otros estudios referenciados, que asocian el nivel social o educativo con patrones más altos de uso, clases más acomodadas o niveles de educación más altos. Es en los entornos más vulnerables donde, sin embargo, la repercusión del consumo de alcohol es mayor, al condicionarse con la presencia de otros factores coadyuvantes nocivos, como problemas legales o accidentes. La explicación por la vulnerabilidad en este caso sería, tanto en hombres como en mujeres, del 70\%, y al categorizar la relación del indicador con la vulnerabilidad con respecto a los demás, este se encontraría en un lugar intermedio, si bien de forma negativa, es decir, a más vulnerabilidad menos consumo en los hombres ${ }^{(25,26,27)}$.

En el caso del consumo de tabaco, las diferencias territoriales son marcadas para el conjunto del indicador, siendo la diferencia significativa entre sexos. La discrepancia es acusada para los hombres, no así para las mujeres. Sin embargo, la vulnerabilidad en los hombres explicaría este comportamiento en el $50 \%$, y en las mujeres prácticamente sería inexplicable por este concepto en el hábito de fumar. No habría un componente de clase social en este caso. Cuando categorizamos la relación con las demás variables estudiadas, el vínculo es de los más bajos, siendo en estos casos las diferencias significativas en los hombres. La literatura también vincula un consumo de tabaco mayor en las clases más bajas. En este caso, el vínculo con la vulnerabilidad aparece explicado en el 50\% para los hombres, con una clara reseña territorial ${ }^{(23)}$.

Finalmente, el grupo de la salud mental, que no presenta diferencias significativas por territorios, ni en mujeres ni en hombres. Sí existen diferencias significativas entre ambos géneros, y aunque en las mujeres la distancia entre territorios más y menos vulnerables existe, esta distancia no presenta significación estadística. El problema se explica con el enfoque de la vulnerabilidad en el $65 \%$ en el caso de las mujeres, y es muy escaso en los hombres. Los vínculos lineales son muy escasos en los hombres, y en las mujeres manejan un $\mathrm{R}^{2}$ de 0,40 . Objetivamente, en los mapas de la ciudad vemos claramente la discrepancia entre hombres y mujeres, y su predominio en estas últimas en los distritos del sur, que son los más vulnerables. Al ordenar las líneas de regresión por sus derivadas, la salud mental en las mujeres aparece entre las más próximas. Encontramos diversos trabajos que vinculan clase social y salud mental, sin desagregación de género. En nuestro caso, el vínculo cuando lo desagregamos es exclusivo de las mujeres, enlazando la vulnerabilidad y la salud mental, así como su explicación en el $65 \%$ de los $\operatorname{casos}^{(29,30,31,32,33)}$.

Como limitaciones de este trabajo, en primer lugar, los datos son autoreferidos para la Encuesta de Salud de la ciudad de Madrid de 2017. Sin embargo, los resultados son muy similares a los que se obtienen de otras encuestas españolas por grupos de edad y sexo, lo que habla en favor de la representatividad del estudio. En segundo lugar, la encuesta incluye 
solamente a población no institucionalizada, por lo que en salud mental puede estar subestimada la prevalencia al no incluirse personas de zonas residenciales. En tercer lugar, la información obtenida puede tener el sesgo de los errores debidos a la tendencia individual a responder lo socialmente más adecuado. En cuarto lugar, este trabajo se ha realizado con población urbana, por lo que los resultados en la población rural pueden ser distintos. Por último, los datos del Indicador de vulnerabilidad proceden de fuentes secundarias de registros poblacionales, y estos se han combinado metodológicamente, por lo que la precisión de las fuentes puede ser distinta.

Las fortalezas de este estudio son que procede de una muestra amplia y de datos poblacionales, así como que las variables en el análisis multivariante han sido controladas con las covariables de sexo, además de su desagregación por este factor en el análisis descriptivo.

A modo de conclusión, existen problemas de salud que claramente se vinculan con el territorio, en los que es posible intervenir desde esta óptica, ya que la organización administrativa y política coloca la visión perceptiblemente en áreas geográficas. Esto permite la desagregación por sexo como realidad e incidir en aquellas situaciones más claramente vinculadas a las mujeres, como son los casos de salud en general, la calidad de vida, la salud mental y la obesidad. Enfrente estarían aquellos más definidos en los hombres como el consumo de alcohol o el de tabaco, con clara relación al territorio marcado por la vulnerabilidad. Por último, estaría el caso del sedentarismo como hábito que por igual marca las zonas de la ciudad de Madrid. Todo ello permite implementar políticas específicas, con recursos tanto económicos como funcionales, así como planificar actividades de prevención y promoción de la salud dirigidas a entornos comunitarios claramente definidos por el territorio.
La selección de indicadores de los principales problemas de salud, y su vinculación a los contextos, barrios y distritos, permite no solamente una planificación más cercana, sino que también se perfila como salida final de unos de los objetivos primarios claros de la epidemiologia moderna: permitir políticas y recursos territoriales definidos, en este caso, para los distritos de la ciudad de Madrid $^{(1,23)}$.

\section{BIBLIOGRAFIA}

1. Marmot MG, Bell R. Social determinants and non-communicable diseases: time for integrated action. BMJ 2019; 364-251. Disponible en https://www.bmj.com/content/364/ bmj.1251 (accedido mayo 2019).

2. Hofrichter R. Health and Social Justice: Politics, Ideology, and Inequity in the Distribution of Disease. 2003.

3. Macintyre S, Ellaway A, Cummins S. Place effects on health: How can we conceptualise, operationalise and measure them. Social Science and Medicine. The Political Ecology of Disease. 2002. 55: 125-139. Disponible en https://pdfs.semanticscholar.org/df1a/22f86d2ea8fd072b0 de3ec99c4c90e13dfac.pdf (accedido abril 2019).

4. Kearns RA, Moon G. From medical to health geography: Novelty, place and theory after a decade of change. 2002. 26-605 Prog Hum Geogr. Disponible en https://pdfs. semanticscholar.org/f6f1/d89d45812ff7000f4badb6903a80fa2482a5.pdf (accedido abril 2019).

5. Malcolm P. Cutchin. The Need for the "New Health Geography" in Epidemiologic Studies of Environment and Health. Health Place. 2007. 725-742.

6. Kane RJ. The ecology of unhealthy places: violence, birthweight, and the importance of territoriality in structurally disadvantaged communities. 2011. Soc Sci Med. 1585-92.

7. Kottow M. Sociología y salud pública: una introducción. Nuevos Folios de Bioética 9 dic 2012. 
8. Rose G. Sick individuals and sick populations. International Journal of Epidemiology.2001; 30: 427-432.

9. Krokstad S, Ernstsen L, Sund ER, Bjørngaard JH, Langhammer A, Midthjell K, Holmen TL, Holmen J, Thoen H, Steinar Westin S. Social and spatial patterns of obesity diffusion over three decades in a Norwegian county population: the HUNT Study. BMC Public Health. 2013; 973. Disponible en https://www.ncbi.nlm.nih.gov/pmc/articles/PMC3853702/. (accedido abril 2019).

10. Zhang Q, Wang Y. Trends in the association between obesity and socioeconomic status in U.S. adults: 1971 to 2000. Obes Res. 2004 .12: 1622-32. Disponible en https:// onlinelibrary.wiley.com/doi/full/10.1038/oby.2004.202. (accedido abril 2019).

11. Sans SG, Paluzie T, Puiga L, Balañá I, Balaguer-Vintró. Prevalencia del consumo de medicamentos en la población adulta de Cataluña. Gac Sanit. 2002 .2.

12. Domínguez-Berjón MF, Borrell C, Cano-Serral G et al. Construcción de un índice de privación a partir de datos censales en grandes ciudades españolas (Proyecto Medea). Gac Sanit., 22 (2008), pp. 179-187

13. Área de Coordinación Territorial y Cooperación Público Social. Metodología para la elaboración del índice de vulnerabilidad territorial de barrios y distritos de Madrid y ranking de vulnerabilidad. 2019. Disponible en: https:// www.madrid.es/UnidadWeb/Contenidos/Publicaciones/ TemaServiciosSociales/IndiceVulnerabil/indicevulnerabilidad.pdf. (accedido mayo 2019).

14. Esteban y Peña MM. Social Obesity and its Determinants 2nd Part as a Discussion from the Social Determinants of the Development: Rent, Education and Mortality. Disponible en: https:/www.ecronicon.com/ ecnu/pdf/ECNU-13-00527.pdf. (accedido may 2019).

15. Madrid City Hall Data Bank. (Accessed 12/2/2020.) Available at: http://www-2.munimadrid.es/CSE6/jsps/menuBancoDatos.jsp.
16. Madrid Salud. Encuesta de Salud 2017 de la ciudad de Madrid. Disponible en http://madridsalud.es/wp-content/ uploads/2019/02/AVANCE-ESTUDIO-SALUD-2018.pdf. (accedido abril 2019).

17. WHOQOL GROUP (1995). The World Health Organization Quality of life assessment (WHOQOL). Position Paper from the World Health Organization. Soc. Sci. Med. 1995. 1.403-09.

18. Sánchez-López MP, Dresch V. The 12-Item General Health Questionnaire (GHQ-12): reliability, external validity and factor structure in the Spanish population. Psicothema. 2008. 20: 839-43.

19. Muñoz PE, Vázquez JL, Rodríguez F, Pastrana E, Varo J. Spanish adaptation of the General Health Questionnaire (GHQ) of Goldberg. Arch Neurobiol 1979. 42: 139-58.

20. Lizán L, Reig A. Cross cultural adaptation of a health related quality of life measurement: the Spanish version of the COOP/WONCA cartoons. Atención Primaria. 1999. 24: 75-82.

21. Lizán L, Reig A, Richart M, Cabrero J. Study on health related quality of life based on information from COOP/ WONCA sheets. Med Clin. 2000. 114: 76-80.

22. WONCA, ERGHO.1995. Measuring functional health status with the COOP/WONCA Charts. Available at: http:// www.globalfamilydoctor.com/publications/publications. asp. (Accesed 3 June 2009).

23. Ministerio de Sanidad Consumo y Bienestar Social. Encuesta Nacional de Salud de España 2017. Disponible en: https://www.mscbs.gob.es/estadEstudios/estadisticas/ encuestaNacional/encuestaNac2017/encuestaResDetall2017.htm. (accedido en mayo 2019).

24. Pacharone Bertolini Bidinotto DN, Pessuto Simonetti J, Mangini Bocchi SC. Men's health: non-communicable chronic diseases and social vulnerability. Rev. Latino-Am. Enfermagem 2016; 24: e 2756 Available in: DOI: http:// dx.doi.org/10.1590/1518-8345.0735.2756. 
25. Landero Hernández R, Villarreal González ME. Consumo de alcohol en estudiantes en relación con el consumo familiar y de los amigos. Psicología y Salud. 2007. 18-23. Disponible en: https://go.galegroup.com/ps/i.do? $\mathrm{p}=\mathrm{AONE} \& \mathrm{sw}=\mathrm{w} \& \mathrm{u}=$ googlescholar $\& \mathrm{v}=2.1 \& \mathrm{it}=\mathrm{r} \& \mathrm{id}=\mathrm{G}$ ALE\%7CA167695811\&sid=classroomWidget\&asid=a7 ec6490. (accedido en mayo 2019).

26. Collins SE. Associations Between Socioeconomic Factors and Alcohol Outcomes. Alcohol Res. 2016. 38: 83-94. Disponible en: https://www.ncbi.nlm.nih.gov/pmc/ articles/PMC4872618/. (accedido en mayo 2019).

27. Karriker-Jaffe KJ, Zemore SE, Mulia N, Jones-Webb R, Bond J, Greenfield TKJ. Neighborhood Disadvantage and Adult Alcohol Outcomes: Differential Risk by Race and Gender. J Stud Alcohol Drugs. 2012. 73: 865-873.

28. Kawachi I, Berkman L. Neighborhoods and health. Oxford University Press. Oxford. 2003.

29. Sampson RJ, Morenoff JD, Gannon-Rowley T. Assessing "neighborhood effects": Social processes and new directions in research. Annual Review of Sociology. 2002. 28: 443-478.
30. Macintyre S, Ellaway A. Neighborhoods and health: An overview. In: Kawachi I, Berkman L, editors. Neighborhoods and Health. Oxford University Press. Oxford: 2003. 20-42.

31. Sánchez-López MP, López-García JJ, Dresch V, Corbalán J. Sociodemographic, psychological and healthrelated factors associated with poor mental health in Spanish women and men in midlife. Women Health. 2008. 48: 445-65.

32. Galea S, Ahern J, Rudenstine S, Wallace Z, Vlahov D. Urban built environment and depression: A multilevel analysis. Journal of Epidemiology and Community Health. 2005. 59: 822-827.

33. Esteban MM, Puerto ML, Fernández Cordero $\mathrm{X}$, Jiménez García R, Gil de Miguel A, Hernández Barrera V. Factores que determinan la mala salud mental en las personas de 16 a 64 años residentes en una gran ciudad. An. Sist. Sanit. Navar. 2012. 35: 229-240. 